\title{
An Improved Technique for Suppression of Intrachannel Four-Wave Mixing in 40-Gb/s Optical Transmission Systems
}

\author{
Hussam G. Batshon, Student Member, IEEE, Ivan B. Djordjevic, Member, IEEE, and \\ Bane V. Vasic, Senior Member, IEEE
}

\begin{abstract}
This letter presents an efficient method for testing the efficiency of modulation techniques for countering the effects of intrachannel four-wave mixing by calculating the sum of all the contributors at a given resonance position (the position affected by the surrounding pulses). In addition to this method, we are proposing a modulation technique that shows a significant $Q$-factor improvement of up to $7.5 \mathrm{~dB}$ (depending on the number of spans and the phase sequence implemented) over the uncoded return-to-zero $\mathrm{ON}-\mathrm{OFF}$ keying. Although the implementation of this phase modulation technique is simple, it is still very efficient in extending the nonlinearity tolerance and enhancing the performance of a high-speed optical transmission system.
\end{abstract}

Index Terms-Communication system nonlinearities, forwarderror correction (FEC), low-density parity-check (LDPC) codes, modulation coding, optical communications, optical Kerr effect.

\section{INTRODUCTION}

$\mathbf{I}$ N HIGH bit-rate return-to-zero ON-OFF keying (RZ-OOK) systems operating at $40 \mathrm{~Gb} / \mathrm{s}$ and above, intrachannel fourwave mixing (IFWM) and intrachannel cross-phase modulation (IXPM) are dominant limiting factors affecting the performance of a high-speed optical transmission system [1]-[5]. Ghost pulses, induced by IFWM, are the result of the interactions of bits (in dispersion-managed systems) satisfying the resonant condition [5]. These interactions cause energy transfers among the pulses resulting in the creation of a spurious pulse in a zero-bit position.

The existing techniques for mitigating this phenomenon previously included: 1) better modulation formats [2]-[4], 2) constrained (line or modulation) coding [5]-[7], 3) equalization [8], [9], 4) optical phase conjugation [10], and 5) finding a proper dispersion mapping [11]. As the creation of ghost pulses is a phase-sensitive effect, the aim for the first approach is to remove short-term phase coherence. Constrained coding prevents the occurrence of most troublesome sequences (the sequences which have a combination of 1's and 0's that rise up the ghost pulse formation) during the encoding process. The linear equalization techniques cannot be used to tackle the nonlinear effects, while the Viterbi equalizer [9] provides the hard decision only, preventing therefore the soft iterative [turbo/low-density parity check (LDPC)] decoding. We have

Manuscript received August 14, 2006; revised November 7, 2006.

The authors are with the Department of Electrical and Computer Engineering, University of Arizona, Tucson, AZ 85721 USA (e-mail: hbatshon@email. arizona.edu; ivan@ece.arizona.edu; vasic@ece.arizona.edu).

Color versions of one or more of the figures in this letter are available online at http://ieeexplore.ieee.org.

Digital Object Identifier 10.1109/LPT.2006.889109 recently shown [5], [9] that the intrachannel nonlinearities can successfully be suppressed by using either combined constrained and forward-error correction (FEC) coding [5] or combined FEC and nonlinear intersymbol interference cancellation based on Bose-Cocke-Jelinek-Raviv algorithm operating a trellis dynamic model of the channel [9]. However, the complexity of those two techniques may be too high for today's available high-speed electronics.

In [5], we have shown that proper choice of initial pulse phases may result in the cancellation of different contributions of ghost pulse creation in bit being in "resonance" (the zero bit position affected by IFWM). The pseudoternary constrained code proposed in [5] is successful in suppression of IFWM, however, it reduces the code rate down to 0.9 .

In this letter, we present an efficient method for suppression of IFWM by choosing the initial pulse phases in such a way to minimize the effect of different ghost-pulse contributors at the zero-bit position being in "resonance" instead of canceling them, without reducing the code rate. We define the new figure of merit for assessing the influence of IFWM, and use it to select a better modulation format among several ones depending on information content. The numerical example combines the method by Gill et al. in [12] $(G)$ and the technique by Zou et al. in [2] (Z). $G$ basically assigns alternating phases of difference $\pi / 2$ to the bits in the original sequence such as $(\pi / 2,0, \pi / 2,0, \ldots)$, while $Z$ applies a fixed eight-bit phase pattern to the sequence. In our IFWM cancellation approach, the information is transmitted by presence or absence of a pulse $(\mathrm{OOK})$, and the parasite phase modulation is used as a means to disturb the phase coherence of the bits being involved in the ghost pulse creation process. The presented modulation technique significantly outperforms $G$ and $Z$ in terms of both $Q$-factor and bit-error rate (BER). ( $Q$-factor improvement over $G$ is up to $2 \mathrm{~dB}$.) The proposed phase modulation combined with an advanced FEC scheme based on LDPC codes provides excellent coding gains.

\section{DESCRIPTION OF AN IFWM CANCELLATION SCHEME}

It was shown in [5] and that proper choice of initial phases may result in suppression of IFWM. The IFWM will be suppressed in any subsequence of length $L, c_{i} c_{i+1}, \ldots, c_{i+L-1}$, if the following complex ghost-pulse constraint is satisfied: for all integers $k, l, m \in\{i, i+L-1\}$ such that $c_{k}=c_{l}=c_{m}=1(k$ and $l$ not necessarily distinct), choose the initial phases $\theta_{k}, \theta_{l}$, and $\theta_{m}$ such that their contribution cancel each other at resonant position $k+l-m$, i.e.,

$$
S=\sum e^{j\left(\theta_{k}+\theta_{l}-\theta_{m}\right)}=0
$$




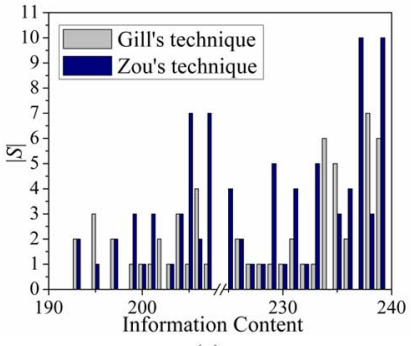

(a)

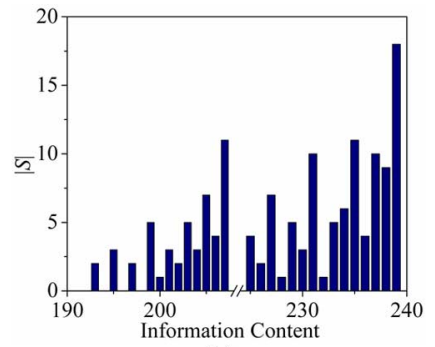

(b)
Fig. 1. Parameter $|S|$ versus the information content, denoted in decimal representation, for different modulation techniques: (a) $G$ and $Z$, (b) the regular RZ-OOK.

where the summation is done over all $(k, l, m) \in\{i, i+L-1\}$ triples for which $c_{k}=c_{l}=c_{m}=1$. Satisfying this constraint is hard to achieve, without sacrificing the code rate (e.g., [5]). Notice that constraint (1) has also to be satisfied in any two successive subsequences of length $L$ as well. So, the idea behind our proposal is to select a simple almost fixed-phase pattern sequence minimizing summation $S$ in (1), and to provide a $Q$-factor improvement over $6.5 \mathrm{~dB}$ which is provided by $G$.

The parameter $S$ in (1) may be used as a figure of merit of a particular modulation format. We may observe a particular zero bit position in a sequence of length $L$, and calculate $S$ for different information content. In Fig. 1, the parameter $|S|$ versus the information content, given in decimal representation (the binary sequence of length $L=9$ is represented as an integer), is compared for three different modulation formats. Only the combinations ranging from 190 to 240 with middle bit being zero are observed; this range has been chosen to demonstrate the general case where $G$ results in a smaller $S$ and some cases where $Z$ minimizes $S$. In Fig. $1, G$ is compared against $Z$ and RZ-OOK.

However, other modulation techniques such as the alternate mark inversion (AMI), might perform better for some specific bit sequences such as the bit sequence "010101010". AMI and similarly duobinary formats assign optical phases to the pulses so that different contributions to ghost pulse creation cancel each other at resonant bit position. Moreover in some other cases, $Z$ might even outperform $G$ such as the bit sequence "111101111" which is considered to be the most troublesome bit sequence in terms of ghost pulse generation. Taking the two bit sequences mentioned above, and testing the summation $|S|$ at the middle bit, the results show that for "010101010" $|S|=$ $0,0,6,6$ and for " 111101111 " $|S|=8,12,12,24$ for $Z$, AMI, $G$, and RZ-OOK, respectively. After all, for a pseudorandom binary sequence (PRBS) input $G$ performs better that the other techniques.

These observations lead us to a conclusion to combine $G$ with $Z$ to form a new phase modulation scheme that further minimizes the sum $S$. The combination mainly depends on finding which scheme among the two gives out the least sum $S$ for a specific 8-bit block of the data sequence and assign it to the bits in the original sequence. The parameter $S$ is precalculated before transmission starts, and the results are stored in a lookup table. Notice that only subsequences for which $Z$ outperforms $G$ are to be stored. Since the general trend is that in most of the cases $G$ is better then $Z$, the length of the lookup table is reasonable short for high-speed implementation. Similar to $G$ and $Z$,

TABLE I

FIBER PARAMETERS

\begin{tabular}{|lcc|}
\hline Parameters & D $_{+}$fiber & D. fiber \\
\hline Dispersion $[\mathrm{ps} /(\mathrm{nm} \mathrm{km})]$ & 20 & -40 \\
Dispersion Slope $\left[\mathrm{ps} /\left(\mathrm{nm}^{2} \mathrm{~km}\right)\right]$ & 0.06 & -0.12 \\
Effective Cross-sectional Area $\left[\mu \mathrm{m}^{2}\right]$ & 110 & 50 \\
Nonlinear refractive index $\left[\mathrm{m}^{2} / \mathrm{W}\right]$ & $2.6 \cdot 10^{-20}$ & $2.6 \cdot 10^{-20}$ \\
Attenuation Coefficient $[\mathrm{dB} / \mathrm{km}]$ & 0.19 & 0.25 \\
\hline
\end{tabular}
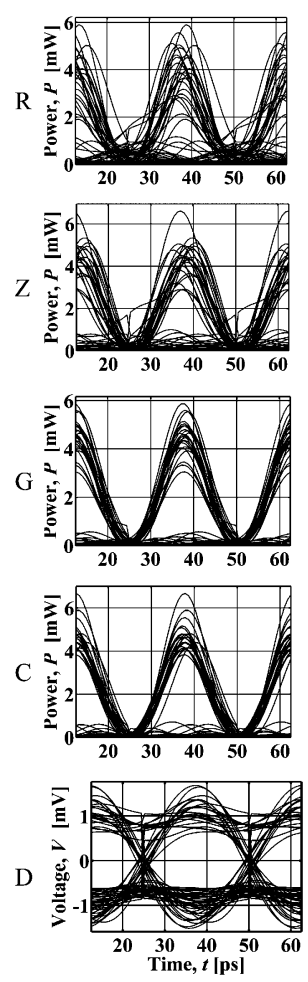

Fig. 2. Eye diagrams (at the output of optical filter) after 35 spans. The columns from left to right: (1) PRBS input using described dispersion map with precompensation of $-800 \mathrm{ps} / \mathrm{nm}$, (2) troublesome input using the same parameters, and (3) PRBS input with precompensation of $-1600 \mathrm{ps} / \mathrm{nm}$. The rows: $(R)$ RZ-OOK, $(Z)$ Zou's technique, $(G)$ Gill's technique, $(C)$ proposed technique, and $(D)$ RZ-DPSK.

the proposed modulation technique requires an additional phase modulator, compared to regular RZ-OOK transmitter.

\section{NUMERICAL RESULTS}

The simulations were run on a realistic dispersion-managed 40-Gb/s single-channel system, and dispersion map is given in [5, Fig. 6]. The span length is set to $L=120 \mathrm{~km}$, and each span consists of $2 L / 3 \mathrm{~km}$ of $D_{+}$fiber followed by $L / 3 \mathrm{~km}$ of $D_{-}$fiber. Precompensation of $-800 \mathrm{ps} / \mathrm{nm}$ and corresponding postcompensation are also applied. The parameters of $D_{+}$and $D_{-}$fibers are as given in Table I. RZ modulation format of a duty cycle of $33 \%$ is observed, and the launched power is set to $0 \mathrm{dBm}$. Erbium-doped fiber amplifiers with noise figure of $5 \mathrm{~dB}$ are deployed after every fiber section, the bandwidth of optical filter is set to $2 R_{b}$ and the bandwidth of electrical filter to $0.65 R_{b}$, with $R_{b}$ being the bit rate $(40 \mathrm{~Gb} / \mathrm{s})$. This dispersion map is selected in such a way that IXPM is low, and IFWM is the predominant effect.

Fig. 2 shows the eye diagrams after 35 spans for two inputs: a regular PRBS input of order 15, and an input that focuses 


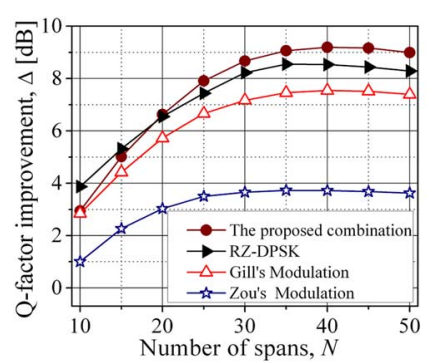

(a)

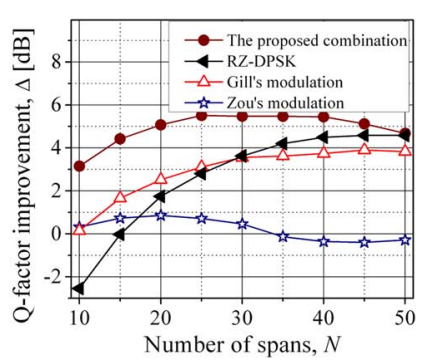

(b)
Fig. 3. Q-factor improvement over the RZ-OOK for (a) PRBS input, and (b) troublesome input.

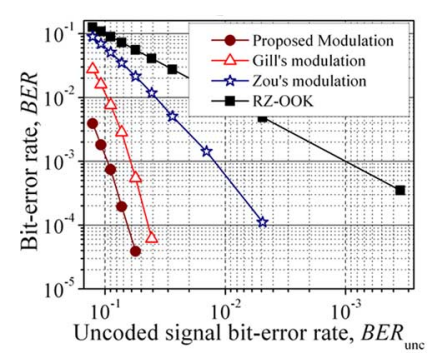

(a)

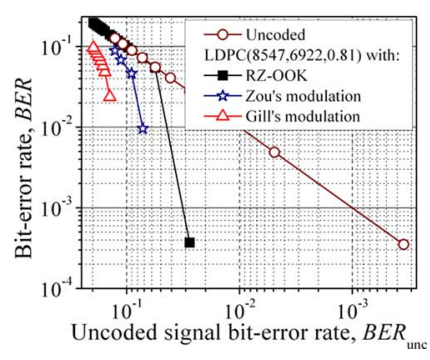

(b)
Fig. 4. BER for different modulation formats for (a) uncoded case, and (b) LDPC coded case.

on the most troublesome blocks observing different modulation schemes of the same length as the PRBS. It is obvious that the eye diagram for the RZ-OOK is highly disturbed, while the eye diagram for proposed modulation format is wide open and the eye diagram for $G$ shows a highly improved eye diagram compared to the RZ-OOK yet not as good as the proposed modulation. This is valid for both types of input. Since the troublesome sequences in the PRBS sequences occur with certain probability, the improvement in the PRBS case is lower.

The same simulator was run while changing the precompensation to $-1600 \mathrm{ps} / \mathrm{nm}$, and the results are shown in Fig. 2. The dispersion map from [11], with the same fiber parameters, was also tested and the improvements were comparable to those of the dispersion map described above.

Fig. 3 shows the $Q$-factor improvement over the RZ-OOK, for $G, Z$, RZ-DPSK, and the proposed modulation technique. It can be noticed that $G$ format achieves the maximum improvement of $7.5 \mathrm{~dB}, Z$ achieves an improvement of $3.8 \mathrm{~dB}$, while $9 \mathrm{~dB}$ is achieved by the proposed modulation technique. The RZ-DPSK with a balanced-receiver, although employing more complicated receiver, performs worse than proposed modulation technique and provides a maximum improvement of $8.5 \mathrm{~dB}$. The improvement for a troublesome input is better than that for regular PRBS input, because for troublesome input $Z$ was employed instead of $G$ resulting in better suppression of ghostpulse effect.

In Fig. 4(a), the proposed modulation technique is compared against $G$ and $Z$ in terms of BER, obtained by counting of errors. As expected, the proposed modulation technique outperforms $G$, and significantly outperforms $Z$.

In Fig. 4(b), the BER performance is shown when LDPC $(8547,6922)$ code of girth-8 (the length of shortest cycle in corresponding bipartite graph), designed as described in [9], is employed in simulations. This LDPC code has a rate of 0.81 , the number of information bits is 6922 , and the codeword length is 8547. Significant performance improvement over LDPC coded RZ-OOK is obtained, with coding gains comparable to that reported in [5] but with lower complexity. To generate the numerical results in Fig. 4, the sequence of length $2^{15}-1$ is transmitted many times over whole transmission systems for different numbers of spans in the range of 10-60, and different amplified spontaneous emission noise realizations. Notice that for the proposed modulation technique supplemented with LDPC code, we were not able to count any errors for number of spans up to 60 . Notice that the simulation results reported here are in very good agreement with VPI TransmissionMaker.

\section{CONCLUSION}

In this letter, the novel figure of merit suitable for testing the influence of IFWM was introduced. Using this figure of merit, the novel modulation technique that selects better modulation format among several ones for sequences being in resonance was proposed. With minimal modifications applied to Gill's method, the method presented herein can result in up to 2-dB improvement in $Q$-factor.

The system performance can be further improved by using more than two different modulation formats, at the expense of increased complexity.

\section{REFERENCES}

[1] A. Mecozzi, C. B. Clausen, and M. Shtaif, "System impact of intrachannel nonlinear effects in highly dispersed optical pulse transmission," IEEE Photon. Technol. Lett., vol. 12, no. 12, pp. 1633-1635, Dec. 2000.

[2] M. Zou, M. Chen, and S. Xie, "Suppression of ghost pulses in 40 Gbps optical transmission systems with fixed-pattern phase modulation," Opt. Express, vol. 13, no. 7, pp. 2251-2255, Feb. 2005.

[3] M. Forzati, J. Martensson, A. Berntson, A. Djupsjobacka, and P. Johannisson, "Reduction of intrachannel four-wave mixing using the alternate-phase RZ modulation format," IEEE Photon. Technol. Lett., vol. 14 , no. 9, pp. $1285-1287$, Sep. 2002

[4] X. Liu et al., "Suppression of intrachannel four-wave-mixing-induced ghost pulses in high-speed transmissions by phase inversion between adjacent marker blocks," Opt. Lett., vol. 27, no. 13, pp. 1177-1179, Jul. 2002.

[5] I. B. Djordjevic and B. Vasic, "Constrained coding techniques for suppression of intrachannel nonlinear effects in high-speed optical transmission," J. Lightw. Technol., vol. 24, no. 1, pp. 411-419, Jan. 2006.

[6] N. Alic and Y. Fainman, "Data-dependent phase coding for suppression of ghost pulses in optical fibers," IEEE Photon. Technol. Lett., vol. 16, no. 4, pp. 1212-1214, Apr. 2004

[7] J. H. Winters, "Equalization in coherent lightwave systems using a fractionally spaced equalizer," J. Lightw. Technol., vol. 8, no. 10, pp. 1487-1491, Oct. 1990.

[8] I. Djordjevic and B. Vasic, "Nonlinear BCJR equalizer for suppression of intrachannel nonlinearities in $40 \mathrm{~Gb} / \mathrm{s}$ optical communications systems," Opt. Express, vol. 14, pp. 4625-4635, May 2006.

[9] B. Vasic, I. B. Djordjevic, and R. Kostuk, "Low-density parity check codes and iterative decoding for long haul optical communication systems," J. Lightw. Technol., vol. 21, no. 2, pp. 438-446, Feb. 2003.

[10] X. Tang and Z. Wu, "Reduction of intrachannel nonlinearity using optical phase conjugation," IEEE Photon. Technol. Lett., vol. 17, no. 9, pp. 1863-1865, Sep. 2005.

[11] A. G. Striegler and B. Schmauss, "Compensation of intrachannel effects in symmetric dispersion-managed transmission systems," $J$. Lightw. Technol., vol. 22, no. 8, pp. 1877-1882, Aug. 2004.

[12] D. M. Gill, X. Liu, X. Wei, S. Banerjee, and W. Su, " $\pi / 2$ alternatephase ON-OFF keyed 40-Gb/s transmission on standard single-mode fiber," IEEE Photon. Technol. Lett., vol. 15, no. 12, pp. 1776-1778, Dec. 2003. 